\title{
Ueber Involutionen höherer Grade.
}

\author{
(Von Herrn Emil Weyr in Prag.)
}

1. Fine Involution $n^{\text {ten }}$ Grades (Strahlen - oder Punktinvolution) ist durch zwei Elementengruppen bestimmt ${ }^{*}$ ). Jede Gruppe besteht aus $n$ Elementen, welche sich gegenseitig involutorisch entsprechen. Sind zwei Involutionen $J_{m}, J_{n}$ bezüglich vom $m^{\text {ten }}$ und $n^{\text {ten }}$ Grade in einer derartigen Beziehung, dass jeder Elementengruppe der einen Involution eine Elementengruppe der anderen entspricht, so nennt man die Involutionen projectivisch.

Sind zwei projectivische Involutionen $J_{m}, J_{n}$ gleichartig (d. h. entweder zwei Strahlen- oder zwei Punktinvolutionen) und befinden sie sich auf demselben Träger (demselben Scheitel oder derselben Geraden), so geschieht es im Allgemeinen $(m+n)$ mal, dass ein Element der einen Involution mit einem der ihm entsprechenden Elemente der anderen Involution zusammenfällt.

Mit anderen Worten: „zwei projektivische Involutionen, welche sich auf demselben Träger befinden, besitzen $(m+n)$ gemeinschaftliche Elemente $\left.{ }^{6 * *}\right)$.

2. Legt man durch den Scheitel einer Strahleninvolution $n^{\text {ten }}$ Grades einen willkürlichen Kegelschnitt $T_{2}$, so schneiden die $n$-strahligen Gruppen der Involution den Kegelschnitt $T_{2}$ in $n$-punktigen Gruppen einer Punktinvolution des $n^{\text {ten }}$ Grades, als deren Träger der Kegelschnitt $T_{2}$ erscheint. Die Punktinvolution auf $T_{2}$ kann in jeder Richtung die Strahleninvolution ersetzen, indem man jede, auf letztere bezügliche Construction mit ersterer vorzunehmen braucht, und dann mittelst des zwischen beiden Involutionen herrschenden einfachen Zusammenhanges der Perspectivität die Resultate zu übertragen hat.

- Ebenso gelangt man von einer Punktinvolution $n^{\text {ten }}$ Grades auf einer Geraden zu einer Tangenteninvolution desselben Grades auf einem Kegelschnitt, welcher dann der Träger der Involution ist.

Es folgt unmittelbar aus dem über Involutionen im Allgemeinen Gesagten, dass auch eine Involution auf einem Kegelschnitte durch zwei Elementengruppen bestimmt erscheint.

*) Siehe Cremona, ebene Curven pag. 27 in der deutschen Ausgabe.

**) ibid. pag. 32 . 
Selbstverständlich genügt es, von den beiden auf Kegelschnitten möglichen Involutionsarten eine zu betrachten, indem mittelst des Gesetzes der Reciprocität die Resulate auch für die zweite Art giltig werden.

3. Sei $\boldsymbol{T}_{2}$ ein Kegelschnitt und Träger einer Punktinvolution $\boldsymbol{n}^{\text {ten }}$ Grades. Die einzelnen $n$-punktigen Gruppen derselben bilden dem Kegelschnitte $T_{2}$ eingeschriebene vollständige $n$-Ecke, von denen jedes $\frac{n(n-1)}{2}$ Seiten besitzt. Wir wollen nun zunächst untersuchen, was für eine Curve die Seiten sämmtlicher dieser $n$-Ecke umhüllen.

Vor Allem ist leicht einzusehen, dass durch jeden Punkt des Trägers $T_{2}(n-1)$ solcher Seiten gehen. Denn jeder Punkt von $T_{2}$ gehört nur einer einzigen Gruppe der Involution an, deren übrige $(n-1)$ Punkte mit ihm verbunden $(n-1)$ durch ihn gehende Tangenten der fraglichen Enveloppe liefern. Es unterliegt keiner Schwierigkeit, zu zeigen, dass durch jeden Punkt der Ebene des Kegelschnittes $T_{2}$ nur $(n-1)$ Tangenten der Enveloppe hindurchgehen, d. h. dass dieselbe eine Curve $(n-1)^{\text {ter }}$ Classe sei.

Es sei der Kegelschnitt $T_{2}$ der Träger der Involution und $p$ ein nicht auf $T_{2}$, aber beliebig in der Ebene von $T_{2}$ gelegener Punkt; $a_{1} a_{2} a_{3} \ldots$ sei eine Gruppe von $n$ Punkten auf $\boldsymbol{T}_{2}$.

Indem man die Punkte $a_{1} a_{2} a_{3} \ldots$ mit $p$ verbindet, erhält man, da jede Verbindungslinie den Träger $T_{2}$ noch einmal schneidet, eine neue $n$-punktige Gruppe $\alpha_{1} \alpha_{2} \alpha_{3} \ldots$ In dieser Art kann man mittelst des Punktes $p$ jeder Gruppe der Involution $(a)$ eine $n$-punktige Gruppe $(\alpha)$ von $T_{2}$ zuordnen, deren Gesammtheit eine neue Involution des $n^{\text {ten }}$ Grades auf $T_{2}$ darstellt.

Die Involution $(a)$ ist offenbar mit der Involution $(\alpha)$ projektivisch, indem jeder Punktgruppe der einen eine Punktgruppe der anderen entspricht.

Beide Involutionen werden somit, da sie vom $n^{\text {ten }}$ Grade sind, $2 n$ gemeinschaftliche Punkte besitzen. Die Berührungspunkte der beiden von $p$ an $T_{2}$ gezogenen Tangenten sind zwei von den gemeinschaftlichen Punkten, wie sofort aus dem perspectivischen Zusammenhange der beiden Involutionen hervorgeht. Ausser diesen giebt es demnach noch $2 n-2$ d. i. $2(n-1)$ weitere gemeinschaftliche Punkte. Von diesen liegen jedoch je zwei auf einer durch $p$ gehenden Geraden. Denn würde z. B. $\alpha_{3}$ mit $a_{1}$ zusammenfallen, so fiele auch gleichzeitig $a_{3}$ mit $\alpha_{1}$ zusammen. Dann liegen aber in einem solchen Falle zwei Punkte $\left(a_{1}\right.$ und $\left.a_{3}\right)$ der Involution $(a)$ mit dem Punkte $p$ in einer Geraden, welche eine Tangente der gesuchten Enveloppe ist. Dies wird, 
weil es 2(n-1) solcher gemeinschaftlichen Punkte giebt, $(n-1)$ mal eintreten, wodurch die von uns aufgestellte Behauptung über die Classe der fraglichen Enveloppe erwiesen ist.

4. Das Resultat der vorhergehenden Betrachtung lässt sich folgendermassen ausdrücken :

„Befindet sich auf einem Kegelschnitte eine Punktinvolution $n^{\text {ten }}$ Grades, so umhïllen die Verbindungslinien entsprechender Punkte eine Curve $(n-1)^{\text {ter }}$ Classe."

Wir wollen diese Curve die Involutionscurve nennen. Ebensoleicht gelangt man zu dem reciproken Satze:

„Befindet sich auf einem Kegelschnitte eine Tangenteninvolution $n^{\text {ten }}$ Grades, so erfüllen die Schnittpunkte entsprechender Tangenten eine Curve $(n-1)^{\text {ter }}$ Ordnung - die Involutionscurve."

Die Involutionscurve einer Involution $n^{\text {ten }}$ Grades an einem Kegelschnitte ist also von der $(n-1)^{\text {ten }}$ Ordnung oder Classe, je nachdem die Involution eine Tangenten - oder Punktinvolution ist.

5. Da man von einer Involution zwei Elementengruppen willkürlich annehmen kann, so liefern die vorigen Ergebnisse die folgenden Sätze:

„Sind einem Kegelschnitte zwei n-Ecke eingeschrieben, so berihtren deren $n(n-1)$ Seiten eine und dieselbe Curve $(n-1)^{\text {ter }}$ Classe. Es giebt dann unendlich viele dem Kegelschnitte eingeschriebene $n-E c k e$, welche dieser Curve umschrieben sind."

Für $n=3$ erhält man den bekannten Satz:

„Sind zwei Dreiecke einem Kegelschnitte eingeschrieben, so sind sie gleichzeitig einem zweiten Kegelschnitte umschrieben. Es giebt dann unendlich viele Dreiecke, welche dem ersteren eingeschrieben und dem zweiten umschrieben sind."

Der letztere Kegelschnitt ist die Involutionscurve der cubischen Punktinvolution, für welche die Ecken der beiden erwähnten Dreiecke zwei Gruppen von Punkten sind.

Für $n=4$ erhält man:

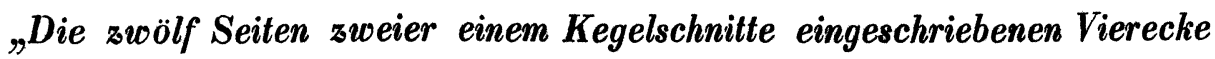
beriihren eine und dieselbe Curve dritter Classe."

Die reciproken Sätze lauten:

,Sind zwei $n$-Seite einem Kegelschnitte umschrieben, so liegen deren $n(n-1)$ Ecken auf einer und derselben Curve $(n-1)^{\text {ter }}$ Ordnung. Es giebt 
dann unendlich viele dem Kegelschnitte umschriebene n-Seite, welche dieser Curve eingeschrieben sind".

„Sind zwei Dreiseite einem Kegelschnitte umschrieben, so sind sie zugleich einem zweiten Kegelschnitte eingeschrieben u.s. w."

„Die zwölf Ecken zweier einem Kegelschnitte umschriebenen Vierseite liegen auf einer und derselben Curve dritter Ordnung u.s. $w$.

6. Befindet sich am Kegelschnitte $T_{2}$ eine Punktinvolution $J_{n} n^{\text {teu }}$ Grades, deren Involutionscurve $C^{n-1}$ ist, so wird man leicht zu jedem Punkte die entsprechenden $(n-1)$ Punkte construiren können. Zieht man nämlich die durch den Punkt gehenden $(n-1)$ Tangenten der Involutionscurve $C^{n-1}$, so wird jede von ihnen den Träger noch einmal und zwar in einem der gesuchten entsprechenden Punkten schneiden.

Umgekehrt schneidet überhaupt jede Tangente der Involutionscurve den Träger $T_{2}$ in zwei entsprechenden Punkten, d. h. in zwei Punkten, welche zu einer und derselben Gruppe gehören. Sollen diese beiden Punkte zusammenfallen, d. h. einen Doppelpunkt der Involution repräsentíren, so muss die Tangente der Involutionscurve, auf welcher sie liegen, zugleich eine Tangente des Trägers $T_{2}$ sein. Da nun der Träger eine Curve der zweiten Classe und die Involutionscurve eine $\operatorname{der}(n-1)^{\text {ten }}$ Classe ist, so besitzen beide $2(n-1)$ gemeinschaftliche Tangenten, welche den Träger $T_{2}$ in den Doppelpunkten der Involution berühren.

„Die 2(n-1) dem Träger $T_{2}$ einer Punktinvolution $n^{\text {ten }}$ Grades und deren Involutionscurve gemeinschaftlichen Tangenten berühren den Träger in den Doppelpunkten der Involution."

Reciprok:

„Die $2(n-1)$ dem Träger $T_{2}$ einer Tangenteninvolution $n^{\text {ten }}$ Grades und deren Involutionscurve gemeinschaftlichen Punkte sind die Beriihrungspunkte der Doppeltangenten der Involution."

7. Jeder der $2(n-1)$ Doppelpunkte einer auf $T_{2}$ befindlichen Punktinvolution $n^{\text {ien }}$ Grades gehört einer Punktgruppe derselben an. Ausser dem Doppelpunkte enthält eine solche Gruppe - eine Doppelpunktsgruppe - noch $(n-2)$ einfache Punkte, welche offenbar die Beschaffenheit haben, dass zwei von den ihnen entsprechenden Punkten im Doppelpunkte zusammenfallen. Es müssen somit auch zwei von den $(n-1)$ aus einem solchen Punkte an die Involutionscurve gehenden Tangenten zusammenfallen, und zwar in der Verbindungslinie des betreffenden Punktes mit dem Doppelpunkte. Daraus folgt 
aber sofort, dass diese Punkte im Allgemeinen der Involutionscurve angehören müssen, d. h. dass es Schnittpunkte des Trägers $T_{2}$ mit der Involutionscurve sind.

„Die (n-2) Punkte einer auf einem Kegelschnitte befindlichen Punktinvolution $n^{\text {ten }}$ Grades, welche mit einem Doppelpunkte einer und derselben Gruppe angehören, sind Schnittpunkte des Trägers mit der Involutionscurve, welche in ihnen die nach dem Doppelpunkte gehenden Strahlen beriihrt."

Da es $2(n-1)$ Doppelpunkte gieht, so giebt es $2(n-1)(n-2)$ solcher Schnittpunkte, und folglich ist die Involutionscurve von der $(n-1)(n-2)^{\text {ten }}$ Ordnung, und daher eine allgemeine Curve $(n-1)^{\text {ter }}$ Classe.

Ebenso reciprok:

„Die $(n-2)$ Tangenten einer auf einem Kegelschnitte befindlichen Tangenteninvolution $n^{\text {ten }}$ Grades, welche mit einer Doppeltangente einer und derselben Gruppe angehören, sind gemeinschaftliche Tangenten des Trägers und der Involutionscurve, welche sie in ihren Schnittpunkten mit der Doppeltangente beriihren."

Die Involutionscurve einer solchen Tangenteninvolution $n^{\text {ten }}$ Grades ist eine allgemeine Curve $(n-1)^{\text {ter }}$ Ordnung.

8. Wir sahen, dass die Involutionscurve einer auf einem Kegelschnitte befindlichen Elementeninvolution durch zwei Elementengruppen bestimmt erschien. Es lässt sich nun zeigen, dass jede Curve von der Familie der Involutionscurve, welche einer Elementengruppe genügt, als Involutionscurve auftreten könne.

Mit anderen Worten:

„Wird einem vollständigen $n-E c k$, welches einem Kegelschnitte $T_{2}$ eingeschrieben ist, eine Curve $C^{n-1}(n-1)^{\text {ter }}$ Classe eingeschrieben, so giebt es unendlich viele $n$-Ecke, velche dem Kegelschnitte eingeschrieben und der Curve $C^{n-1}$ umschrieben sind, d. h. die Curve $C^{n-1}$ kann als die Curve einer auf $T_{2}$ befindlichen Punktinvolution $n^{\text {ten }}$ Grades betrachtet werden.".

Denn zieht man von einem beliebigen Punkte $x_{1}$ des Kegelschnittes $\boldsymbol{T}_{2}$ an $C^{n-1}$ die $(n-1)$ Tangenten, so werden diese auf $T_{2}$ die Punkte $x_{2} x_{3} \ldots x_{n}$ bestimmen. Wenn man nun die $n$ Punkte $(x)$ als eine Gruppe einer auf $T_{2}$ befindlichen Punktinvolution $n^{\text {ten }}$ Grades betrachtet, für welche die $n$ Ecken des $n$-Ecks, welchem $C^{n-1}$ eingeschrieben wurde, eine zweite Gruppe bilden, so ist dadurch die Involution und folglich auch eine Involutionscurve bestimmt, welche von derselben Classe wie $C^{n-1}$ mit dieser die Seiten des dem Kegel- 
schnitte $T_{2}$ eingeschriebenen $n$-Ecks und die $(n-1)$ von $x_{1}$ nach $x_{2} x_{3} \ldots x_{n}$ gehenden Strahlen zu gemeinschaftlichen Tangenten besitzt.

Die Gesammtzahl dieser gemeinschaftlichen Tangenten beträgt somit $\frac{n(n-1)}{2}+(n-1)$, d. i. $\frac{(n-1)(n+2)}{2}$, also genau so viel Tangenten, als zur Bestimmung einer Curve $(n-1)^{\text {ter }}$ Classe nothwendig sind. Die beiden betrachteten Curven werden also nur dann von einander verschieden sein können, wenn die gemeinschaftlichen $\frac{(n-1)(n+2)}{2}$ Tangenten zu einem System von gemeinschaftlichen Tangenten zweier Curven $(n-1)^{\text {ter }}$ Classe gehören.

Dies ist jedoch nicht der Fall. Denn da je $(n-1)$ von diesen Tangenten durch einen Punkt hindurchgehen, so müssten die übrigen eine Curve $(n-2)^{\text {ter }}$ Classe berülren, was aber aus dem nämlichen Grunde unmöglich ist, weil von ihnen abermals je $(n-1)$ durch einen Punkt gehen, und diese Punkte daher der Curve $(n-2)^{\text {ter }}$ Classe als Bestandtheile erster Classe angehören müssten. Nun giebt es $\frac{n(n-1)}{2}$ solcher Punkte, welche keine Curve $(n-2)^{\text {ter }}$ Classe darstellen können.

Die Curve $C^{n-1}$ muss demnach mit der Involutionscurve identisch sein, wodurch unsere Behauptung erwiesen ist. Das reciproke Ergebniss lautet.

„Wird einem vollständigen $n$-Seit, welches einem Kegelschnitte $T_{2}$ umgeschrieben ist, eine Cure $(n-1)^{\text {ter }}$ Ordnung umgeschrieben, so giebt es unendlich viele $n$-Seite, welche dem Kegelschnitte umgeschrieben und der Curve eingeschrieben sind; $d . h$. die Curve kann als die Curve einer auf $T_{2}$ befindlichen Tangenteninvolution $n^{\text {ten }}$ Grades betrachtet werden."

Das vollständige $n$-Seit liefert für die im letzten Satze besprochene Involutionscurve $\frac{n(n-1)}{2}$ Punkte. Da eine Curve $(n-1)^{\text {ter }}$ Ordnung durch $\frac{(n-1)(n+2)}{2}$ Punkte bestimmt erscheint, so kann man von der Involutionscurve überdies $(n-1)$ Punkte willkürlich annehmen. Nun liefert jedes Paar - entsprechender Tangenten der Involution einen Punkt der Involutionscurve, woraus wir folgendes Ergebniss ziehen:

"Eine Involution $n^{\text {ten }}$ Grades ist vollkommen bestimmt, sobald man eine Gruppe von Elementen und weitere $(n-1)$ Elementenpaare kennt."

9. Wird von einer auf dem Kegelschnitte $T_{2}$ zu bestimmenden Tangenteninvolution $\boldsymbol{n}^{\text {ten }}$ Grades eine $n$-elementige Gruppe angenommen, so bildet diese ein vollständiges dem Träger umschriebenes $n$-Seit, dessen $\frac{n(n-1)}{2}$ 
Ecken Punkte der Involutionscurve sind. Die Involution selbst und mit ihr die Involutionscurve wird erst durch Annahme einer zweiten $n$-elementigen Gruppe bestimmt sein. Nimmt man von dieser zweiten Gruppe nur $(n-1)$ Tangenten an, so ist die Involution noch unbestimmt und wird erst durch Hinzufügen einer Tangente zu diesen $(n-1)$ Tangenten bestimmt sein. Lässt man diese letzte Tangente variabel, so erhält man unendlich viele Involutionen, welche eine $n$-elementige und eine $(n-1)$-elementige Gruppe gemein haben.

Die Involutionscurven werden demgemäss die $\frac{n(n-1)}{2}$ Ecken des durch erstere Gruppe gebildeten $n$-Seits und die $\frac{(n-1)(n-2)}{2}$ Ecken des durch die zweite Gruppe gebildeten (n-1)-Seits gemeinschaftlich haben. Da die Involutionscurven von der $(n-1)^{\text {ten }}$ Ordnung sind, so werden je zwei von ihnen $(n-1)^{2}$ gemeinschaftliche Punkte haben. Nun ist $\frac{n(n-1)}{2}+\frac{(n-1)(n-2)}{2}=(n-1)^{2}$, und folglich bilden die Ecken des besprochenen $n$-Seits und $(n-1)$-Seits die sämmtlichen Durchschnittspunkte aller der betrachteten Involutionscurven. Da wir die $n$-elementige und die $(n-1)$-elementige Gruppe beliebig annehmen konnten, so ziehen wir hieraus folgenden Satz:

„Die Ecken eines vollständigen einem Kegelschnitte umschriebenen nSeits mit den Ecken eines demselben Kegelschnitte umschriebenen (n-1)-Seits stellen ein System von Durchschnittspunkten zweier Curven $(n-1)^{\text {ter }}$ Ordnung dar."

Ebenso:

„Die Seiten eines vollständigen einem Kegelschnitte eingeschriebenen nEcks mit den Seiten eines demselben Kegelschnitte eingeschriebenen (n-1)-Ecks stellen ein System von gemeinschaftlichen Tangenten zweier Curven $(n-1)^{\text {ter }}$ Classe dar."

10. Es werden also nach dem ersten Satze des vorigen Artikels die sechs Ecken eines einem Kegelschnitte umschriebenen Vierseits mit den drei Ecken eines demselben Kegelschnitte umschriebenen Dreiseits neun Durchschnittspunkte zweier Curven dritter Ordnung darstellen.

Hieraus fliesst eine besonders einfache Construction des neunten Scheitels eines Curvenbüschels dritter Ordnung, wenn sechs Scheitel die Ecken eines vollständigen Vierseits bilden. Construirt man nämlich von den beiden dem Vierseit nicht angehörenden Scheiteln die zwei Tangenten an jenen dem Vierseit eingeschriebenen Kegelschnitt, welcher die Verbindungslinie dieser 
zwei Scheitel berührt, so schneiden sich die beiden Tangenten in dem neunten Scheitel.

11. Befindet sich auf einem Kegelschnitte $T_{2}$ eine Punktinvolution $n^{\text {ten }}$ Grades neben einer solchen $\operatorname{des} m^{\text {ten }}$ Grades, so wird es eine leicht bestimmbare Anzahl von Punktepaaren geben, welche sowohl der einen als auch der anderen Involution angehören.

Die Involution $\boldsymbol{n}^{\text {ten }}$ Grades besitzt eine Involutionscurve $(n-1)^{\text {ter }}$ Classe, deren Tangenten auf $\boldsymbol{T}_{2}$ Punktepaare dieser Involution bestimmen. Die Involutionscurve der zweiten Involution ist von der $(m-1)^{\text {ten }}$ Classe. Beide Curven haben demnach $(n-1)(m-1)$ gemeinschaftliche Tangenten, welche auf $T_{2}$ ebenso viele Punktepaare bestimmen, die beiden Involutionen angehören ihnen gemeinschaftlich sind. Daher der Satz:

"Zwei Involutionen auf demselben Träger resp. vom $m^{\text {ten }}$ und $n^{\text {ten }}$ Grade besitzen $(n-1)(m-1)$ gemeinschaftliche Elementenpaare."

In der Betrachtung des 3. Artikels war $m=2$.

Prag, im Januar 1870. 